\title{
Dual-modal Assessment for in Vivo Investigation of Neurovascular Characteristic of Cerebral Edema Induced by Lipopolysaccharide
}

\section{Weitao Li}

Nanjing University of Aeronautics and Astronautics

\section{Yameng Zhang}

Nanjing University of Aeronautics and Astronautics

Qian Xie

Nanjing Medical University

Yamin Yang

Nanjing University of Aeronautics and Astronautics

Liuye Yao

Nanjing University of Aeronautics and Astronautics

Xue Ning

Nanjing University of Aeronautics and Astronautics

zhiyu Qian ( $\nabla$ yamengzhang@njit.edu.cn )

Nanjing University of Aeronautics and Astronautics

\section{Research}

Keywords: neurovascular characteristics, optical-electrical joint system, neuro-electrophysiology, spectrum signal, laser speckle contrast imaging

Posted Date: November 1st, 2021

DOl: https://doi.org/10.21203/rs.3.rs-944735/v1

License: (a) (1) This work is licensed under a Creative Commons Attribution 4.0 International License. Read Full License 


\section{Dual-modal assessment for in vivo investigation of neurovascular characteristic of cerebral edema induced by lipopolysaccharide}

Weitao $\mathrm{Li}^{1} \triangle$, Yameng Zhang ${ }^{1,2} \Delta$, Qian $\mathrm{Xie}^{3}$, Yamin Yang ${ }^{1}$, Liuye Yao ${ }^{1}$, Xue Ning ${ }^{1}$, Zhiyu Qian $^{1 *}$

${ }^{1}$ Department of Biomedical Engineering, Nanjing University of Aeronautics and Astronautics, 211106, No. 169 Sheng Tai West Road, Jiang Ning District, Nanjing, China;

${ }^{2}$ Department of Computer Engineering, Nanjing Institute of Technology, No.1 Hongjing Avenue, 211167, Nanjing, China;

${ }^{3}$ School of Pharmacy, Nanjing Medical University, 101 Longmian Avenue, 211166, Nanjing, China

\section{ABSTRACT:}

The pathological features of cerebral edema are complicated, but usually only intracranial pressure (ICP) is regarded as the most important indicator for monitoring cerebral edema. The research focused on investigating the neurovascular characteristic of the lipopolysaccharide (LPS)-induced cerebral edema model in rats by using simultaneous electrophysical and hemodynamic recording. The results showed that neurophysiology (firing rate (FR), interval histogram index (ISI), and the power spectrum of local field potential (LFPs power)) and hemodynamic response (oxygenated hemoglobin $\left(\mathrm{HbO}_{2}\right)$, deoxyhemoglobin $(\mathrm{HbR})$ and relative cerebral blood flow (CBF)) were linearly related, and the Pearson's correlation coefficient was determined by the BBB integrity after LPS injection. Furtherly, the improvement of ${ }^{*}$ Corresponding authors: Zhiyu Qian, Department of Biomedical Engineering, Nanjing University of Aeronautics and Astronautics, Email: zhiyu@nuaa.edu.cn

$\triangle$ co-authors: These authors contributed equally to this work first 
treatment after two agents were observed successfully through these neurophysiological and hemodynamic parameters. The optical-electrical joint method provided a technical solution for cerebral edema functional monitoring and anti-edema drug efficacy evaluation. Our findings revealed the neurovascular and BBB impact of cerebral edema and improved the limitation of in vivo pathological diagnosis of cerebral edema.

KEY WORDS: neurovascular characteristics; optical-electrical joint system; neuroelectrophysiology; spectrum signal; laser speckle contrast imaging

\section{INTRODUCRION}

Cerebral edema is a potentially fatal disorder which is present with many common cerebral pathologies, such as stroke, traumatic brain injury, central nervous system tumors, brain ischemia, and intracerebral hemorrhage ${ }^{1}$. Previous studies have been devoted on unraveling the clinical and pathological features of cerebral edema and evaluating the therapeutic effects of various drugs for cerebral edema by the value of $\mathrm{ICP}^{2}$. However, it is difficult to effectively distinguish the pathological characteristics of cerebral edema and examine the effects of anti-edema drugs merely based on the direct measurement of ICP.

As the normal brain function is facilitated by a tight coupling between neuronal activity, cerebral blood flow, and metabolism, neurovascular function highlights the intimate relationships between neurons, neuroglia and vascular cells ${ }^{3}$. The alterations of neurovascular function can be closely related to the neurological disease state and 
the impairment of neurovascular function was found during brain disorders including cerebral edema. For example, Matilde et al. found that subarachnoid hemorrhage could result in acute changes in the cerebral microcirculation ${ }^{4}$. The monitoring of neuronal activity, blood oxygen and cerebral blood flow is thus necessary and helpful for understanding the fundamental pathophysiology of edema development as well as for identifying possible therapeutic targets of cerebral edema. For example, Brad et al. monitored that brain activity and the time-dependent neurophysiological and hemodynamic response during the cerebral ischaemia and reperfusion process ${ }^{5}$.

One of the key mechanisms underlying the formation of cerebral edema is the disruption of the blood brain barrier (BBB), which plays an essential role in maintaining the balance of water-electrolyte distribution inside and outside the neurons and glia 6 . In the meantime, a large body of evidence has indicated that BBB is the core structure for modulating the brain circulation and neurovascular function ${ }^{7}$. Nevertheless, few studies have investigated the pathological characteristics of cerebral edema from the perspective of the neural activity ${ }^{8}$. Therefore, we herein aim to elucidate the possible relationship between neuronal and hemodynamic parameters so as to bridge the complexity of BBB during the progression of cerebral edema. In addition, the use of LPS in the experiment was taken into consideration that LPS could activate the expression of inflammatory factors, such as IL-I $\beta$, TNF- $\alpha$, et al., the factors could initiate or contribute to the BBB injury and eventually cause the formation of cerebral edema ${ }^{9}$.

The aim of this paper is to systematically explore the neural activity and hemodynamic 
response during the progression of LPS induced by cerebral edema and to evaluate the effects of anti-edema drugs. The optical-electrical joint system was developed to record electrophysiological and hemodynamic parameters concurrently and the assessment method can feedback the neurovascular and BBB characteristic of cerebral edema from the term of neural activity and hemodynamic response, which enabled providing a potential technical solution for clinical cerebral edema pathological development monitoring and drug efficacy evaluation.

\section{MATERIALS AND METHODS}

\section{Animal preparation}

All in vivo animal experiments were conducted in accordance with the guidelines of the Institutional Animal Care and Use Committee at Nanjing University of Aeronautics and Astronautics. The research was approved by institutional animal care and Use committee of Nanjing Medical university (IACUC-1909023). Female Sprague Dawley rats (140-180 g) from Animal Experiment Center of Nanjing Medical University (Nanjing, China) were housed in cages with food and water ad libitum. All rats were maintained in a room with a $12 \mathrm{~h}$ light/dark cycle and allowed a 3-day adaptation prior to experiments. The rats were randomly assigned to 6 groups, approximately 10 in each group and the experiment procedure for each group is listed in Table.1. The rats in G1 and G2 groups were injected intravenously with LPS and saline respectively at the beginning of the experiment. Afterwards, the data were recorded every 20 minutes till 140 min after the injection. The rats in the G3, G4, and G5 groups were injected 
with mannitol (MA), hypertonic saline (HS), and saline respectively at 120 min after the LPS injection, and afterwards the corresponding data was recorded every 20 min till 220 min. The rats injected with saline during the entire process (G6) were used as control. In the experimental recording process, the rats were kept under a moderately anesthetized state, and the heart rate was at approximately $350 \mathrm{bpm}$.

Table.1 Group of experimental design

\begin{tabular}{ccc}
\hline groups & Dose [b.w.] & $\begin{array}{c}\text { Total recording } \\
\text { time(minutes })\end{array}$ \\
\hline G1 & $1 \%$ LPS $9 \mathrm{mg} / \mathrm{kg}$ & 140 \\
G2 & $0.9 \%$ saline $1 \mathrm{ml} / \mathrm{kg}$ & 140 \\
G3 & $1 \%$ LPS $9 \mathrm{mg} / \mathrm{kg}+20 \% \mathrm{MA} 2 \mathrm{~g} / \mathrm{kg}$ & $220\left(2^{\text {nd }}\right.$ injection, 120) \\
G4 & $1 \%$ LPS $9 \mathrm{mg} / \mathrm{kg}+7.5 \% \mathrm{HS} 4 \mathrm{ml} / \mathrm{kg}$ & $220\left(2^{\text {nd }}\right.$ injection, 120$)$ \\
G5 & $1 \%$ LPS $9 \mathrm{mg} / \mathrm{kg}+0.9 \%$ saline $1 \mathrm{ml} / \mathrm{kg}$ & $220\left(2^{\text {nd }}\right.$ injection, 120$)$ \\
G6 & $0.9 \%$ saline $1 \mathrm{ml} / \mathrm{kg}+0.9 \%$ saline $1 \mathrm{ml} / \mathrm{kg}$ & $220\left(2^{\text {nd }}\right.$ injection, 120$)$ \\
\hline
\end{tabular}

\section{Surgical procedures}

Surgical procedures were performed after animals were deeply anesthetized with $10 \%$ pentobarbital $(165 \mathrm{mg} / \mathrm{kg})$. The animal anesthesia and operation procedures were described previously in details ${ }^{10}$. Briefly, the cranial hole was roughly 1.0 to $-1.0 \mathrm{~mm}$ posterior to bregma and 1.0 $2.0 \mathrm{~mm}$ lateral to the midline for neural signal recording (A-P:1.0 1.0 mm; L:1.0 $2.0 \mathrm{~mm}$ ). Meanwhile, the cranial window was opened on the ipsilateral brain, and the coordinates is -1.0 to $-4.0 \mathrm{~mm}$ posterior to bregma and 
$0 \sim 3.0 \mathrm{~mm}$ lateral to the midline for spectrum acquisition and laser speckle contrast imaging (A-P: $-1.0 \sim-4.0 \mathrm{~mm}$; L:0 $3.0 \mathrm{~mm}$ ).

\section{Dual-modal System setup and recording procedures}

The schematic representation of our multimodal system is shown in Fig. 1 for recording electrophysical and hemodynamic parameters, including spikes, $\mathrm{CBF}, \mathrm{HbO}_{2}$ and $\mathrm{HbR}$. Briefly, the integrated optical-electrical monitoring platform is composed of the neuroelectrophysiology recording system, the intrinsic optical signal (IOS) spectrum acquisition system, and the laser speckle imaging platform. For avoiding the influence of laser on electrophysiological signal collection, the laser speckle contrast images were obtained immediately after the electrophysiological signals were recorded for 5 minutes.

(a)

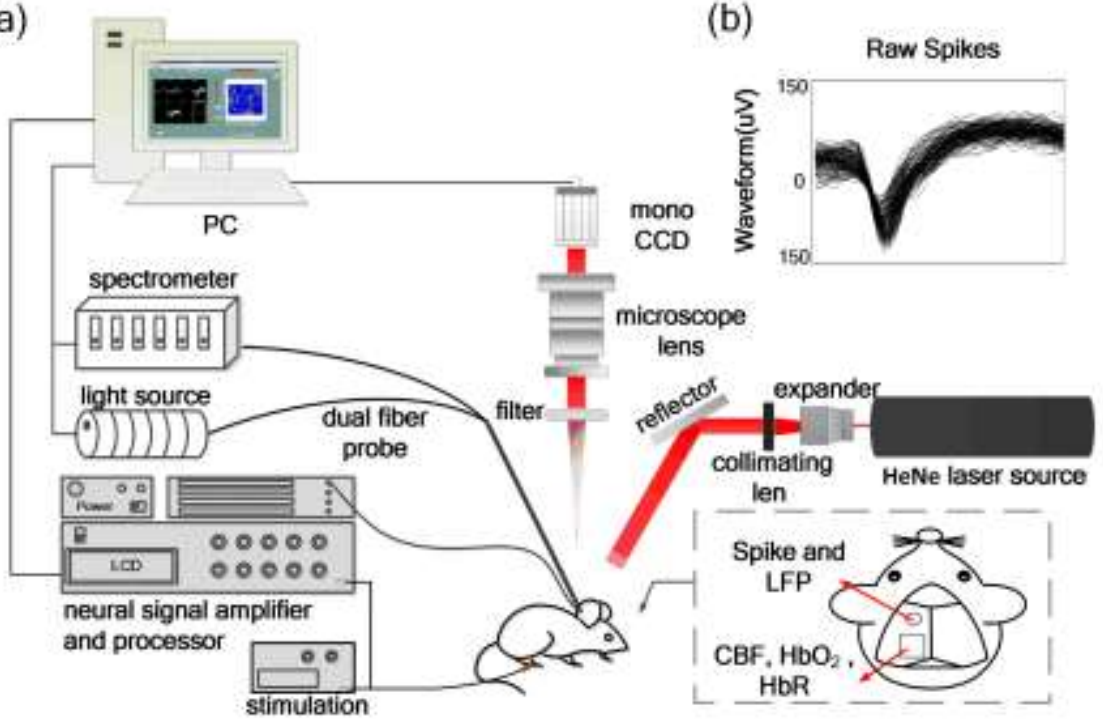

(c)

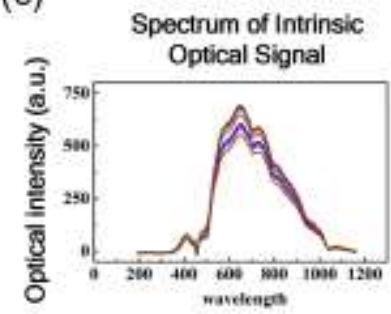

(d) Raw speckle image

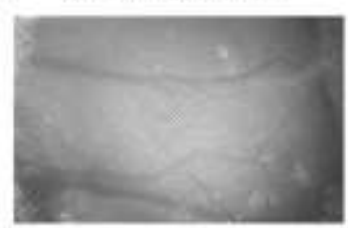

Fig. 1 (a) Schematic diagram of the optical-electrical joint monitoring system, (b) Representative electrophysiological raw spikes, (c) Raw spectrum of intrinsic optical signal, and (d) Pseudo-color map of blood flow.

\section{Spikes and local field potentials (LFPs) recording}

The rats were implanted with a $2 \times 2$ microelectrode array (diameter about $33 \mu \mathrm{m}$, 
impedance more than $1 \mathrm{M} \Omega$ ) targeting the somatosensory motor region. The arrays were inserted at a depth of approximately $600 \mu \mathrm{m}$ below the surface of somatosensory cortex, where the largest cortical spikes and LFPs amplitudes were obtained. Four-channel LFPs and spikes were recorded simultaneously from the rat hippocampus by Cerebus Acqusition System (Blackrock Microsystems, USA). LFPs were amplified (gain: 4000), filtered $(0-250 \mathrm{~Hz})$ and sampled at $2000 \mathrm{~Hz}$. Meanwhile, spikes (high pass filter: $0.25-5 \mathrm{kHz}$, sampled at $30 \mathrm{kHz}$ ) with power of more than 4.5 times the standard deviation from the baseline mean were extracted and stored with the time stamps per channel. The inter-spike interval histogram (ISI) is defined as the conditional probability of a first spike at time $t_{n+1}$ after a spike at time $t_{n}$, seen in Eq.1.

$$
I S I(n)=t_{n+1}-t_{n}
$$

Here, $t_{n}$ is the firing time of the $\mathrm{n}$ th spike, and is the firing time of the $\mathrm{n} t h+1$ spike. Then the mean ISI was calculated during $0.2 \mathrm{~s}$ epoch after stimulation and regarded as an indicator of spike coding pattern.

The detailed LFPs collection and theta power analysis process were previously introduced in our earlier study ${ }^{11}$. For better comparison, an external electrical signal (5 mA) with fixed frequency $(0.1 \mathrm{~Hz})$ and duration (300 s) was applied by stimulating the left forepaw of the rats during the recording process as reference. The spikes were identified from the raw data and clustered by K-means method. Baseline drifts were removed, and various typical frequencies, including delta $(0.5-4 \mathrm{~Hz})$, theta $(4-8 \mathrm{~Hz})$, alpha $(8-16 \mathrm{~Hz})$, beta $(16-30 \mathrm{~Hz})$, and gamma $(30-60 \mathrm{~Hz})$ band, were obtained through LFPs processing after wavelet transform. 


\section{Blood oxygen saturation measurement}

Broadband spectrum was recorded from the in-house built spectrum measurement apparatus. A dual-fiber probe consisted of two optical fibers with the diameter of 200 $\mu \mathrm{m}$ and core-to-core distance of $200 \mu \mathrm{m}$ was used to collect the optical signals. The excitation light with the wavelength from $200 \sim 1100 \mathrm{~nm}$ was coupled into one of the optical fibers. Then the light scattered back from the samples was collected by the other optical fiber and was detected by a spectrometer (USB 2000, Ocean Optics, USA). The obtained raw spectrum data were converted into changes of $\mathrm{HbO}_{2}$ and $\mathrm{HbR}$ by the IOS analysis, which was carried out by the modified Beer-Lambert law ${ }^{12}$ and least squares, seen in Eq.2.

$$
\Delta A=\varepsilon I \Delta C=\log _{10} \frac{I_{a}}{I_{0}}
$$

where $\varepsilon$ is the molar absorptivity, $l$ is the path length factor, $\Delta C$ is the change in molar concentration compared to baseline measurement, $l_{a}$ is the post light intensity, and $I_{0}$ is the baseline light intensity. Molar absorptivity and path length factor values were obtained from previous studies reported by Kuboyama et al. ${ }^{13}$. The concentrations of both $\mathrm{HbO}_{2}$ and $\mathrm{HbR}$ can be distinguished by applying least squares to the following relationship, seen in Eq.3.

$$
\begin{aligned}
& \Delta A(\lambda)=\varepsilon_{\mathrm{HbO}_{2}}(\lambda) \Delta c_{\mathrm{HbO}_{2}} D_{a}(\lambda)+\varepsilon_{\text {HbR }}(\lambda) \Delta c_{\text {HbR }} D_{a}(\lambda)+\varepsilon_{\text {Cytaаз } D}(\lambda) \Delta c_{\text {Cytaаa-D }} D_{a}(\lambda) \\
& +\varepsilon_{\text {Cytc-D }}(\lambda) \Delta c_{\text {CytCDD }} D_{a}(\lambda)+\varepsilon_{F A D}(\lambda) \Delta c_{F A D} D_{a}(\lambda)+\mu_{s}^{\prime}(\lambda) \Delta s D_{s}(\lambda)
\end{aligned}
$$

Here, $\varepsilon_{\mathrm{HbO} \mathrm{O}_{2}}, \varepsilon_{\mathrm{HbR}}, \varepsilon_{\text {Cytaa3-D }}, \varepsilon_{\mathrm{CytC}-D}$ and $\varepsilon_{F A D}$ are corresponding molar absorptivity of $\mathrm{HbO}_{2}, \mathrm{HbR}$, Cytc, Cytaa3 and FAD, respectively. $\Delta c_{\mathrm{HbO}_{2}}, \Delta c_{\mathrm{HbR}}, \Delta c_{\text {Cytaa3-D }}, \Delta c_{\text {cytc-D }}$ and $\Delta C_{F A D}$ are corresponding change in molar concentration respectively. Given that 
the absorption intensity of the tissue is related to the scattering characteristics, the scattering coefficient should be regarded as another tissue chromophore. $D_{a}(\lambda)$ was the ratio of absorbance to absorption coefficient, $D_{s}(\lambda)$ was the ratio of absorbance to scattering coefficient and $\Delta s$ was the relative change of chromophore scattering. The following six wavelengths of light $450 \mathrm{~nm}, 470 \mathrm{~nm}, 500 \mathrm{~nm}, 550 \mathrm{~nm}, 570 \mathrm{~nm}$ and $600 \mathrm{~nm}$ were selected in consideration of the appropriate absorption peaks and equivalent absorption points of $\mathrm{HbO}_{2}, \mathrm{HbR}$, Cytaa3-D, Cytc-D and $\mathrm{FAD}$, which can be regarded as the temporal response for $\mathrm{HbO}_{2}$ and $\mathrm{HbR}$.

\section{Brain blood flow observation using laser speckle contrast imaging}

Laser speckle contrast imaging was employed to visualize the changes of brain blood flow during the entire experimental stages. In details, a He-Ne laser was adjusted by using a collimator (632.8 $\mathrm{nm}$ and $15 \mathrm{~mW}$, Thorlabs, USA). Then the laser passed an expander and irradiated the rat brain region with a diameter of $\sim 12 \mathrm{~mm}$ with an incident angle of $30^{\circ}-45^{\circ}$. The illuminated region was magnified through a zoom stereo microscope (50486A, Navitar, USA), and the raw speckle images were captured through a monochrome CCD camera (12 bit, Point Grey, Canada) with $2448 \times 2048$ pixels. The exposure time of the CCD was set as $20 \mathrm{~ms}$, and the images were acquired through the programming software at $20 \mathrm{~Hz}$. Then, a stack of 30 raw images were stored and processed by using Matlab (Mathworks, MA). The following equation Eq.4 was used to calculate speckle contrast $(K)$.

$$
K=\frac{\delta}{<I>}
$$

where $\delta$ was the standard deviation and $<I>$ was the mean of intensities within a 
sliding window. Here, a $5 \times 5$ pixels spatial window was used as the convolution window. The CBF was calculated in Eq.5 with the above sliding window, and the detailed algorithm was followed by our previous study and introduced earlier ${ }^{14}$.

$$
C B F=\frac{1}{\tau}=\beta \frac{1}{T K^{2}}
$$

The speckle contrast value at each pixel of the captured image was calculated to obtain the velocity distribution map, showing the pattern of blood flow on the cortical surface. For quantitative evaluation, regions of interest (ROIs) of CBF were selected in LSCl images and the CBF had a running average of $1 \mathrm{~s}$ before analysis of changes in response to LSP injection as baseline.

\section{Brain water content measurement}

At the end of the experiment, all rats were sacrificed by decapitation under the deep anesthesia state, and the brains were removed immediately. The cortical tissues (about $1 \mathrm{~mm}^{3}$ in volume) was extracted and placed on the electronic analytical balance to measure the wet weight (WW). The dry weight (DW) was measured after the brain tissue was dried for $24 \mathrm{~h}$ above $100{ }^{\circ} \mathrm{C}$. The percent of brain water content was obtained from the Eq. 6

$$
\text { Water content }(\%)=(W W-D W) \times 100 \% \text { WW }
$$

\section{Immunohistochemistry analysis}

In this study, IL-1 $\beta$, TNF- $\alpha$ and IgG were used to evaluate the pathological characteristics of cerebral edema by ELISA kit. The detailed experimental operation was introduced earlier literature ${ }^{15}$. The Optical Density (O.D.) at $450 \mathrm{~nm}$ could be distinguish using a spectrometer. 


\section{Statistics analysis}

Statistical analysis was performed using SPSS software (SPSS Statistics v. 19.0, IBM Corp, Armonk, USA) by one sample t test, one-way analysis of variance (ANOVA) test and repeated measures ANOVA followed by Dunnet's post-hoc test. Differences were considered to be statistically significant at $p<0.05$ and $p<0.01$. For correlation analysis, Pearson correlation coefficient was determined between two variables. $p<0.05$ was considered statistically significant.

\section{RESULTS}

In Fig.2, Representative cerebral blood flow, original spectral and the electrophysical response of the superficial cortex from individual sample were collected by the opticalelectrical joint system in vivo sequentially. In correspond to the map of blood flow, G1 group clearly displayed a noticeable blood flow velocity increase in the in Fig.2(a), where the relative blood flow of the G2 group remained stable in Fig.2 (b). Similarly in the original spectral analysis, the spectral peak of G1 group has dropped significantly, while G2 kept constant in Fig.2 (c) and Fig.2 (d). In addition, in the electrophysical signal recording, the left forepaw of the rat was stimulated using an electrical signal. As shown in Fig.2 (e-j), the counts of spikes in the cortex region were the highest at $0.06 \sim 0.08 \mathrm{~s}$ after the electrical stimulation, and the firing frequency in the ipsilateral cortical SI region reached 16 . This trend in the PSTH lasted approximately $40 \mathrm{~min}$ after the LPS injection. Then the peak of spikes has slightly changed, The maximum counts of spikes in the rat were in the interval of $0.14 \sim 0.16 \mathrm{~s}$, and the highest firing 
frequency was decreased to 8 , which showed that the exponential decline trend of peristimulus time histogram (PSTH) gradually disappeared after 40 minutes. The timefrequency analysis also suggested that the injection of LPS rendered a significant decrease in the power spectrum of the low frequency band after 80 minutes. Noted that the acceleration of local cerebral blood flow, the inhibition of neuron activities as reflective of electrophysical signal changes and irregular neural spike pattern coexisted due to the LPS injection, suggested that a strong neurovascular coupling between cerebral neural activity and hemodynamic response may synchronously occur during the LPS induced edema process and proved the validity of the opticalelectrical joint system.

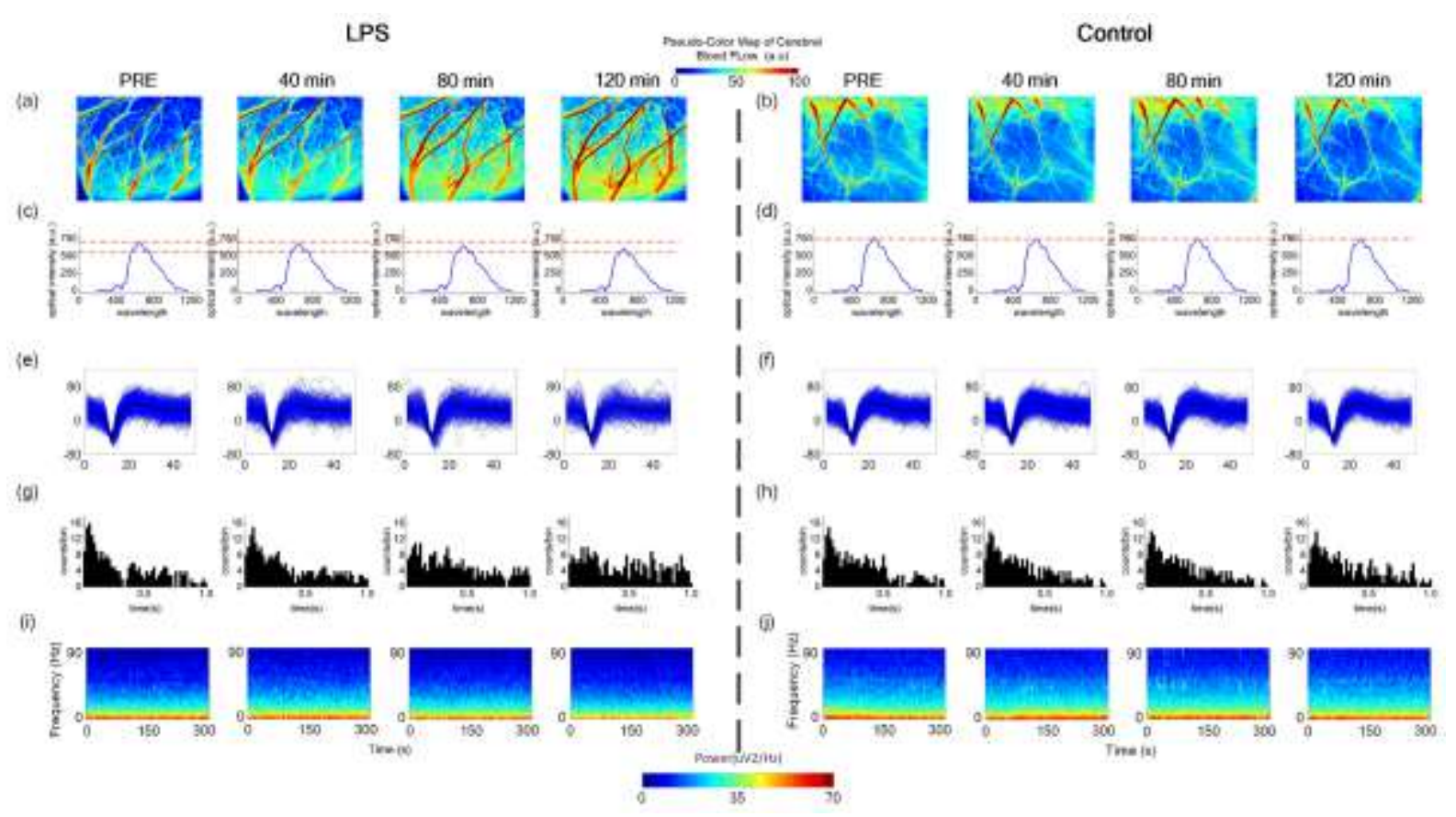

Fig.2 Representative CBF in the superficial cortex in the (a) G1and (b) G2 group, representative spectrum of the same cortex in the (c) G1 and (d) G2 group, representative spikes waveforms of the ipsilateral cortical in the (e) G1 and (f) G2 group, peristimulus time histogram (PSTH) the ipsilateral cortical in the (g) G1 and (h) G2 group, time-frequency analysis in the (i) G1 and (j) G2 group, Each data was 
recorded in vivo pre- and post-injection respectively.

In consideration of the physiological difference between different rats, the calculated CBF and blood oxygen were normalized, and averaged data in the control group before any injection was used for normalization. As shown in Fig.3 (a), the CBF in the control group remained stable over time, while the CBF in the LPS-treated group gradually increased after the LPS injection and continued to rise up to $1.035 \pm 0.112$ at 140 min $(P<0.01)$. Fig.3 (b) compared the $\mathrm{HbO}_{2}$ relative variation among the LPS and control group, The level of $\mathrm{HbO}_{2}$ decreased significantly in the LPS-treated group $(P<0.05)$ after 60 min post treatment, and continued to decrease to $0.761 \pm 0.106$ at 140 min $(P<0.01)$. In contrast to the changes of $\mathrm{HbO}_{2}$, Fig.3 (c) revealed that the $\mathrm{HbR}$ in the LPS group rose after $60 \mathrm{~min}$ and reached $1.306 \pm 0.108$ at $140 \mathrm{~min}(P<0.01)$.

Similarly, the spikes and LFPs data were also normalized and analyzed. In Fig.3 (e) - (f), mean FR and mean ISI of the LPS group barely changed at the early stage of LPS treatment (0-40 min). From 60 to $140 \mathrm{~min}$, mean FR and ISI gradually declined and eventually reached to $0.60 \pm 0.092$ and $0.41 \pm 0.112(P<0.01, P<0.01)$, respectively. Besides, Fig.3 (f) revealed the theta spectrum of LFPs in the Ipsilateral cortical region over time. In the first 20 to 60 min, LFPs power in the LPS group remained stable in general. Yet in the last $80 \mathrm{~min}$, the frequency spectrum of the LPS group began to decline, and especially at the end of the experiment, the frequency spectrum decreased to $33.12 \pm 5.903 \mu \mathrm{V}^{2} / \mathrm{Hz}$, with statistical difference $(P<0.01)$. In summary, the results of FR, ISI, and LFPs power showed significant downward trends after 60 min of LPS injection, which represented the time-dependent inhibition of 
electrophysical activities and abnormal neural functions in the SI region of the rat brain.

(a)

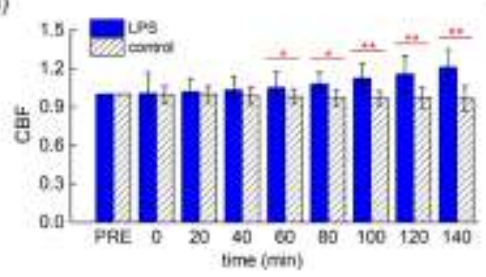

(d)

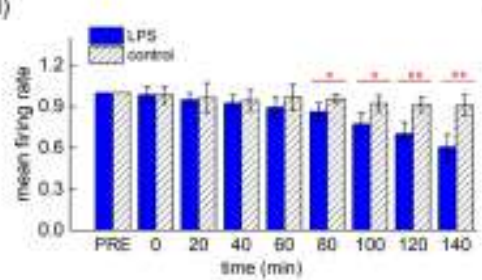

(b)

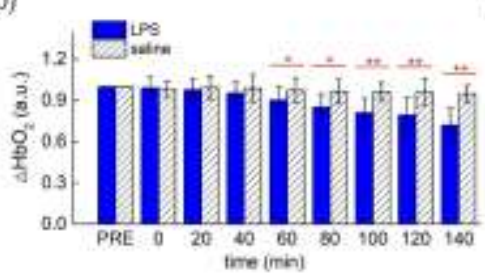

(e)

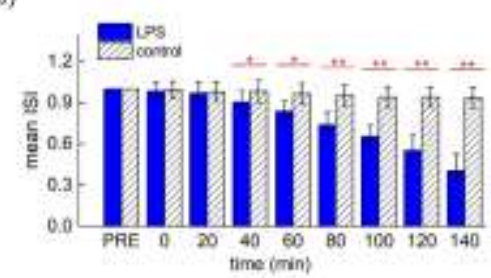

(c)

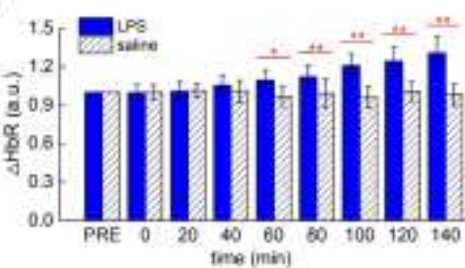

(f)

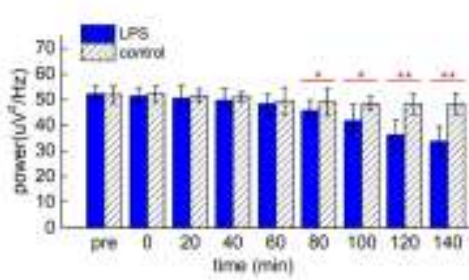

Fig.3 (a) Mean $\mathrm{CBF}$, (b) mean $\mathrm{HbO}_{2}$, (c) mean deoxygenated hemoglobin $\mathrm{HbR}$, (d) mean FR, (e) mean ISI and (f) mean theta LFPs power calculated at each time point pre- and post-injection respectively. All data was recorded every 20 min from the starting point ( $0 \mathrm{~min}$ ) to $140 \mathrm{~min}$ after LPS intravenous injection and the data acquisition process lasted for 5 min for each recording (Error bars $=S E M .{ }^{*} P<0.05$;

$$
{ }^{* *} P<0.01 \text { compared with control group) }
$$

After the LPS-induced cerebral edema model in rat was successfully established and the corresponding changes in both hemodynamic and neuronal responses were identified, then the therapeutic efficiency of MA and HS in treating cerebral edema were further investigated. As depicted in Fig.4 (a) - (c), the variation in blood flow and blood oxygen of brain edema induced by LPS were consistent with those in Fig. 3 within the first time period of 0-140 min. Notably, compared to the sustainable growth in the G5 group, i.e., treated with $0.9 \%$ saline, both the CBF and $\mathrm{HbR}$ levels of the MA group (G3) and $\mathrm{HS}$ group (G4) gradually decreased and approached to the state of control group (G6) within 120 min after the drug injection. Besides, the levels of CBF and $\mathrm{HbR}$ in the G3, G4 and G5 groups were compared at the end of recording by post hoc test, which demonstrated statistical significance $(P=0.0035, P=0.0043$ and $P=0.0012, P=0.0019)$. The levels of 
$\mathrm{HbO}_{2}$ of the $\mathrm{G} 3$ and $\mathrm{G} 4$ groups also slowly increased and nearly reached the state of control group (G6), which was statistically significant compared with the G5 group $(P=0.0038, P=0.0041)$.

The variations of corresponding spikes and LFPs in the cerebral edema model upon various treatments were illustrated in Fig.4 (d)-(f). Before the injection of the MA, HS, and $0.9 \%$ saline, the changes in FR, ISI and theta power in LPS-treated groups (G3, G4, and G5 group) were consistent with those in Fig.3. To our interests, without any drug intervention, the FR and ISI of the G5 group continued to decrease and eventually dropped to $0.392 \pm 0.1097$ and $0.390 \pm 0.1080$. Yet, less drop in the FR and ISI were noticed in the G3 and G4 groups (FR: $0.596 \pm 0.1004,0.675 \pm 0.1006$ and ISI : $0.620 \pm 0.1009,0.691 \pm 0.1096$ respectively), possibly due to the injection of MA and HS. In addition, the LFPs power also showed a similar trend, where both MA and HS might prevent the exacerbation of the cerebral edema. The difference in LFPs power between G3, G4 and G5 groups at 220 min ( $P=0.0045, P=0.0042)$ was statistically significant. Taken together, the above results indicated that hemodynamic responses to LPS might appear earlier than the neuronal response in terms of electrical signal changes. 
(a)

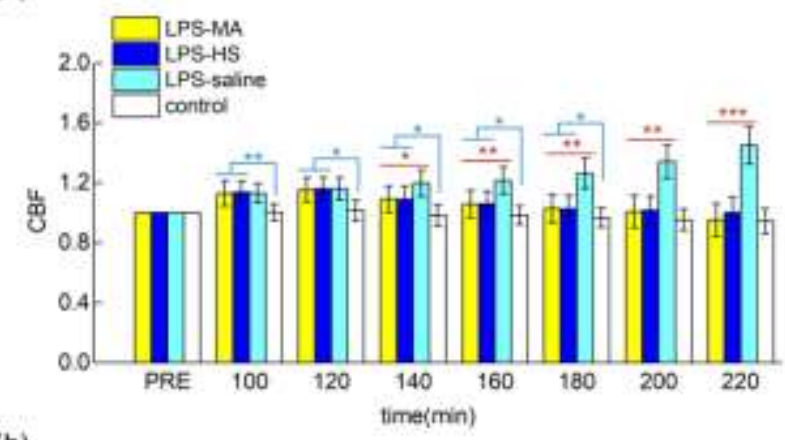

(b)

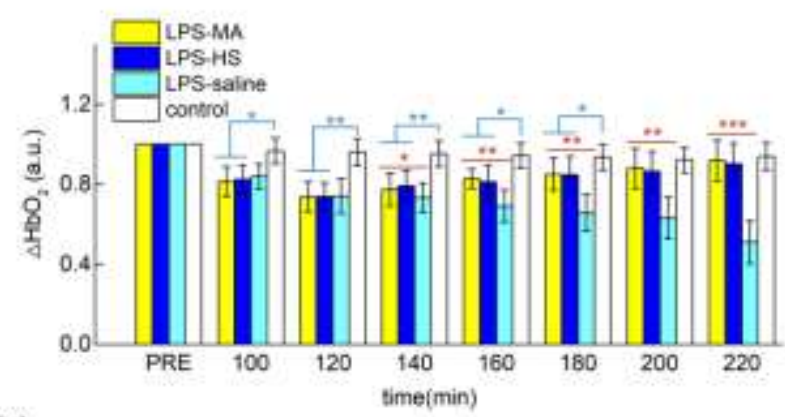

(c)

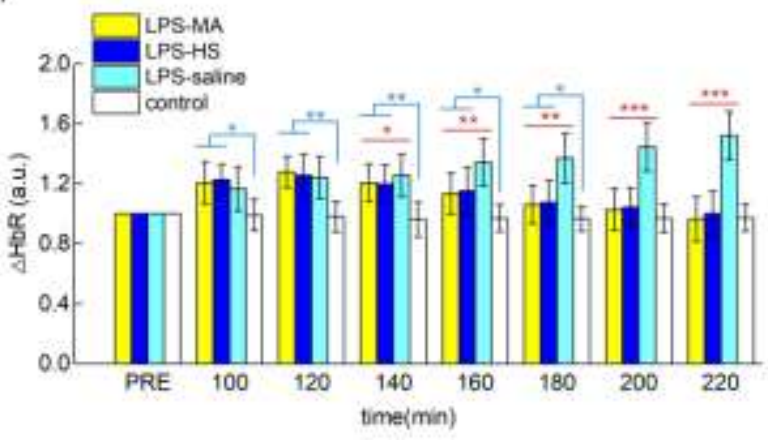

(d)

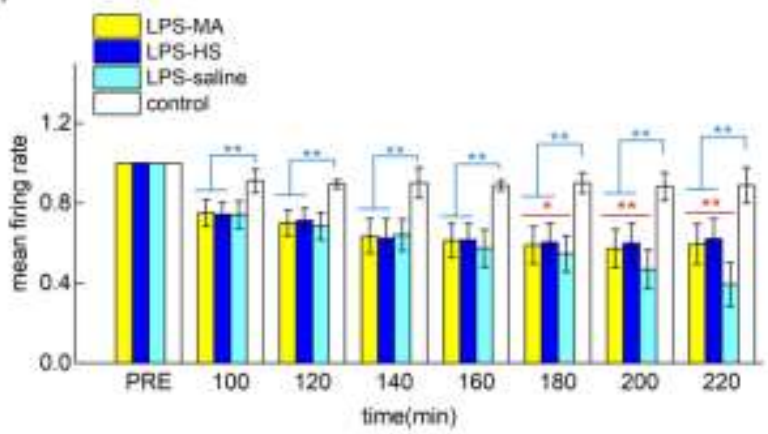

(e)

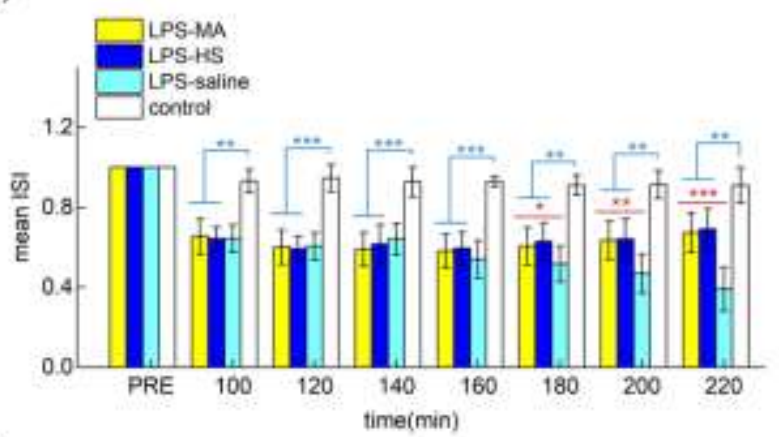

(f)

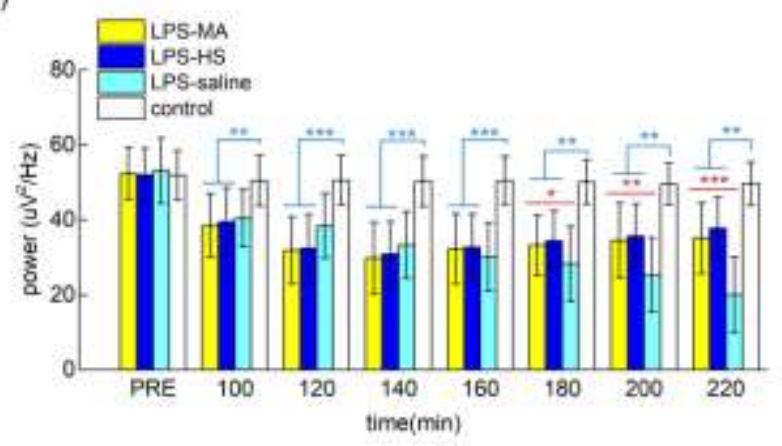

Fig.4 (a) Mean CBF, (b) mean $\mathrm{HbO}_{2}$, (c) mean $\mathrm{HbR}$, (d) mean FR, (e) mean ISI and $(f)$ mean theta LFPs power in different treatment and control conditions respectively. All data was recorded every 20 minutes from the $100 \mathrm{~min}$ to $220 \mathrm{~min}$ since the therapeutic agent was injected at the time point of $120 \mathrm{~min}$. The data acquisition process lasted for $5 \mathrm{~min}$ at each time point. (Error bars $=S E M$. Red marked ${ }^{*} P<0.05$, red marked ${ }^{*} P<0.01$ compared with LPS-saline group. Blue marked ${ }^{*} P<0.05$, blue marked ${ }^{* *} P<0.01$, blue marked ${ }^{* * *} P<0.01$ compared with control group without any treatment)

To further identify the underlying mechanism of the therapeutic effects of MA and HS on LPS-induced cerebral edema, cortical tissues in each group were collected and stained with Evans Blue dye, which can only extravasate into leaked blood-brain barrier. Fig.5 (a) 
showed Evans Blue staining images of rat brains in each group. Obviously, Evans Blue yielded a deeper penetration in G5 of which rats did not receive any therapeutic intervention, and no obvious difference was found between the G3, G4 group, Besides, the faliure of Evans Blue staining infiltration in the G6 group indicated BBB integrity. Furthermore, the ICP value, the water content of the brain tissues and the concentrations of multiple inflammatory mediators including IL-1 $1 \beta$, TNF- $\alpha$ and IgG in different groups were analyzed shown in Fig.5 (b-f). The G5 group rendered the highest ICP, water content and the highest concentrations of all tested inflammatory factors as compared to other groups, which indicated that LPS indeed induced the accumulation of a large amount of water and inflammatory mediators. In addition, the ICP, the water content and the concentrations of inflammatory mediators in the G3 and G4 treatment groups were significantly lower than those in the G5 group, yet higher than the normal condition, which demonstrated the consistency of the optical-electrical joint system in evaluating the therapeutic effects of MA and HS from the biochemical side.

(a)

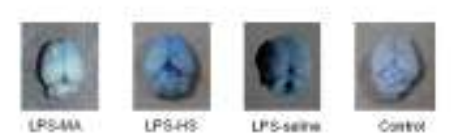

(b)

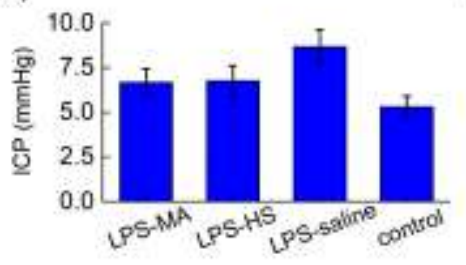

(c)

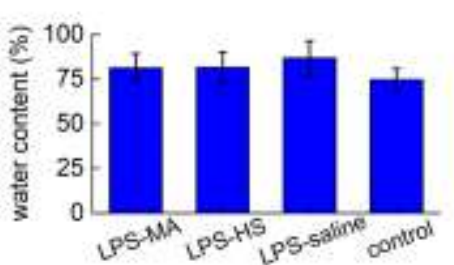

(d)

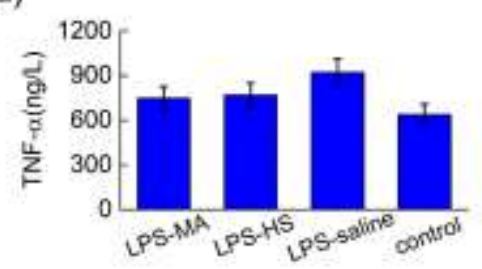

(e)

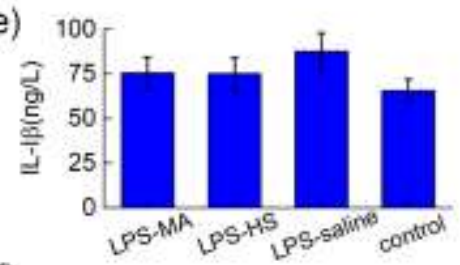

(f)

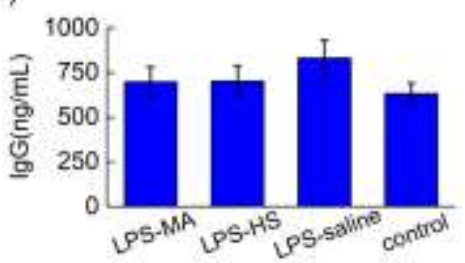

Fig.5 Therapeutic Effects on LPS-induced cerebral edema model. Comparison of (a) the Evans blue staining tissue, (b) intracranial pressure, (c) cerebral water content, (d) inflammatory factor TNF- $\alpha$, (e) inflammatory factor IL-I $\beta$ and (f) 
globulin IgG in different treatment and control conditions, respectively.

In order to determine whether the LPS-induced cerebral edema can change the neurovascular characteristics, the correlation between neuronal and hemodynamic response was analyzed at different time points during the experimental process between LPS and control group. According to the ANOVA's test result of the LPS group, the periods of the correlation analysis was divided into 0-120 minutes and 140-220 minutes. Fig.6 (a) revealed that the decline in the FR was closely correlated with the increase in CBF and HbR (Pearson's $r=-0.647, R^{2}=0.420$; Pearson's $r=-0.802, R^{2}=$ 0.628), while the FR produced a positive correlation with the changes of $\mathrm{HbO}_{2}$ (Pearson's $r=0.812, R^{2}=0.643$ ) within $0-120$ minutes. After 120 minutes, interestingly, both $\mathrm{R}^{2}$ and the Pearson's coefficient significantly declined by $50 \%$, especially in terms of CBF (Pearson's $r=-0.317, R^{2}=0.0597$ ) and HbR (Pearson's $R=-0.286, R^{2}=0.041$ ) in Fig.6 (b). In conclusion, the Pearson's coefficient difference between Fig.6 (a) and Fig.6(b) showed a varying relationship of neurovascular characteristics during the LPS induction time compared to control group in Fig.6(c). 
(a)
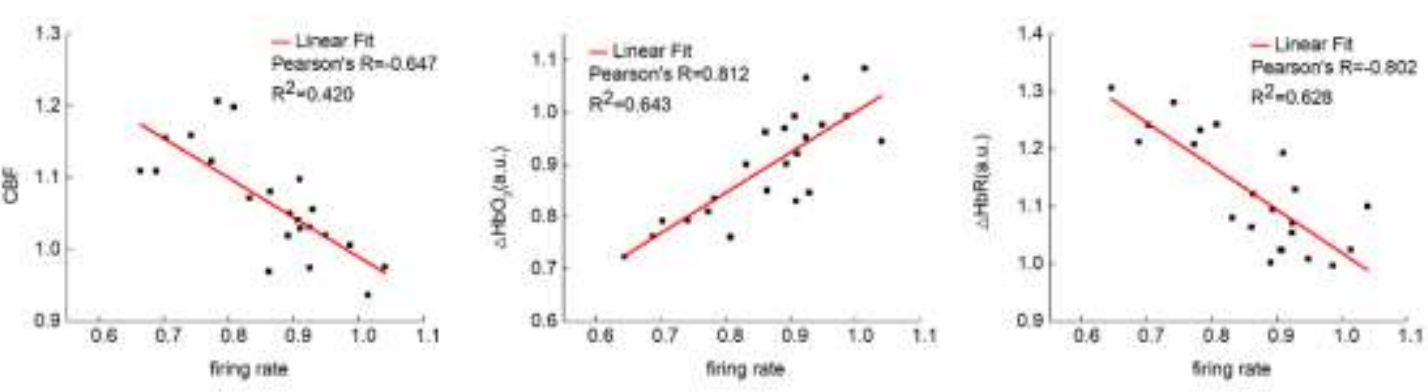

(b)
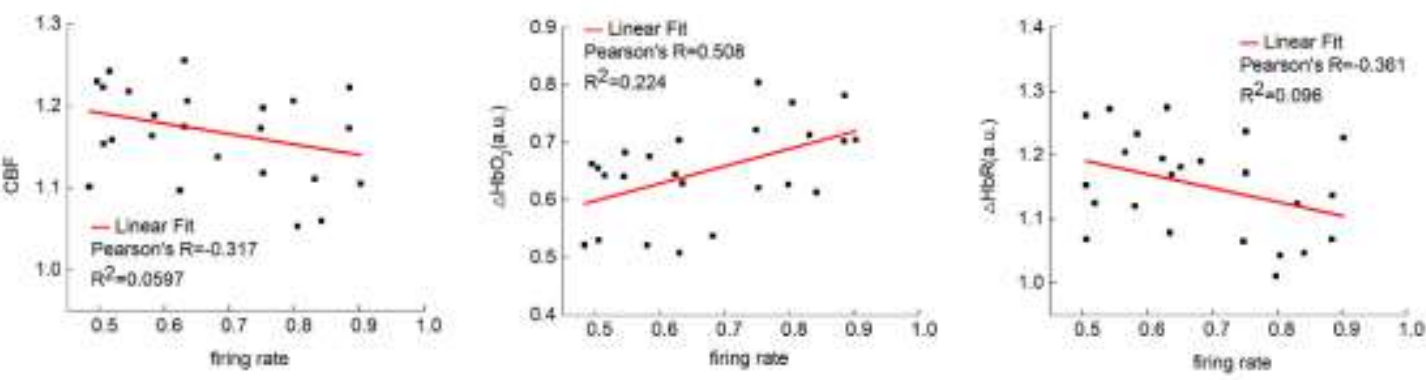

(c)
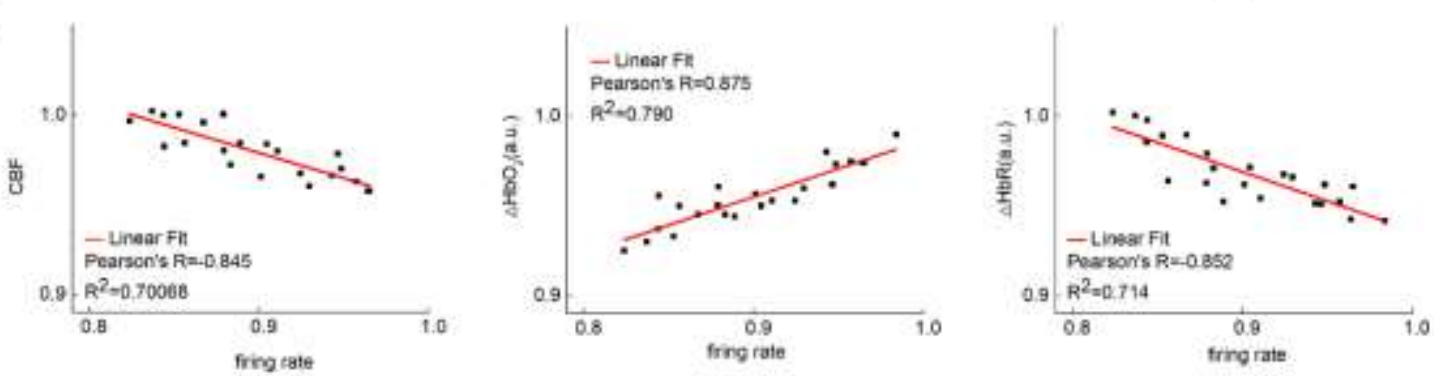

Fig. 6 Correlation between hemodynamic and neuronal activity in the LPSinduced model. (a) Pearson correlation between mean firing rate and $\mathrm{CBF}, \mathrm{HbO}_{2}$, or HbR after LPS injection within 0-120 min, respectively. (b) Pearson correlation between mean firing rate and $\mathrm{CBF}, \mathrm{HbO}_{2}$, or $\mathrm{HbR}$ after LPS injection within 140220 min, respectively. (c) Pearson correlation between mean firing rate and CBF, $\mathrm{HbO2}$, or HbR of control within 0-220 min, respectively

In the further research of the relationship of neurovascular characteristics between neural and blood flow parameters, the determination coefficient $R^{2}$ of Pearson correlation maps between hemodynamic and neuronal activity were shown in Fig.7. Comparing the only LPS induction time of $0-120$ minutes and $140-220$ minutes, the determination coefficient $R^{2}$ between the electrophysiological parameters (FR, ISI, power) and hemodynamic parameters $\left(\mathrm{CBF}, \mathrm{HbO}_{2}, \mathrm{HbR}\right)$ were significantly 
reduced in Fig.7(a) and 7(b), which suggested LPS induction time exerted impact on the confidence and satisfaction of the Pearson correlation. Notably as shown in Fig.7(c) and 7 (d), the determination coefficient $R^{2}$ of the G3 and G4 groups presented an obvious trend of rising, and the determination coefficient $R^{2}$ of the G4 group has returned to state of $\mathrm{G} 1$ group, which indicating the fitting degree of Pearson correlation between hemodynamic and neuronal activity gradually recovered, so treatment groups can alleviate the condition of cerebral edema especially HS treatment group. Above all, the determination coefficient $R^{2}$ of Pearson correlation between neural and hemodynamic characteristics could reflect the progression of pathological features of LPS induced cerebral edema and monitor drug efficacy in vivo, which is consistent with the experimental results of inflammatory factors and ICP in Fig.5. 
(a)

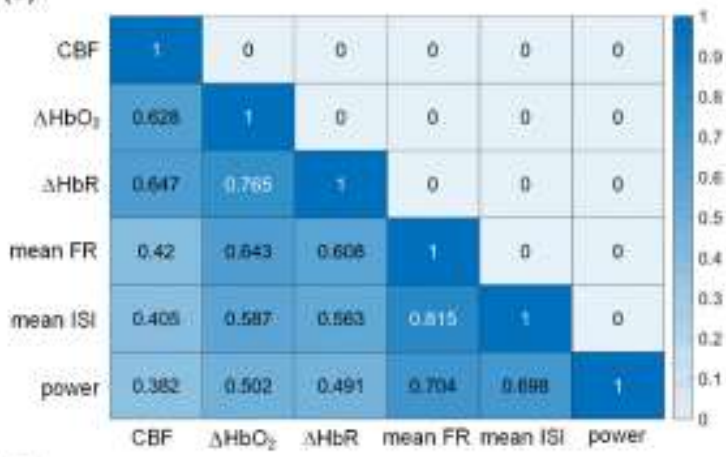

(c)

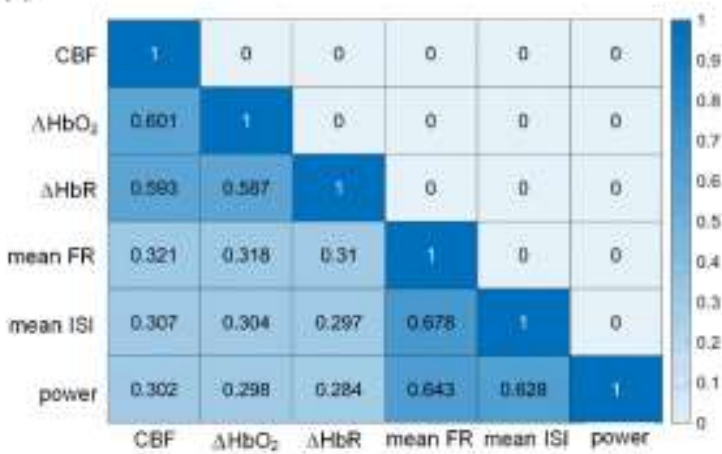

(e)

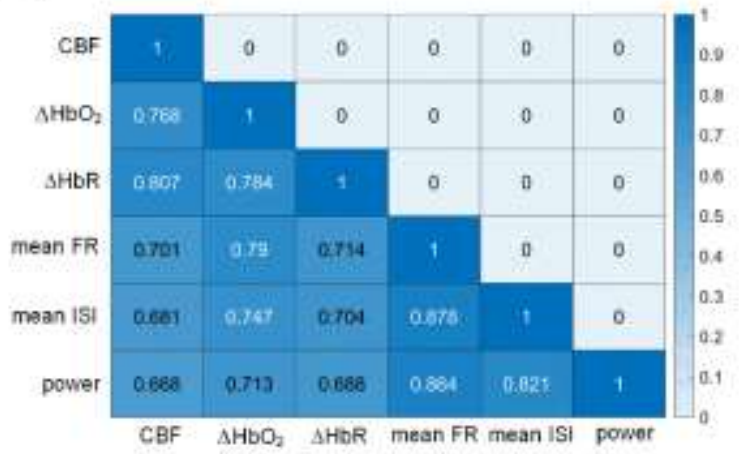

(b)

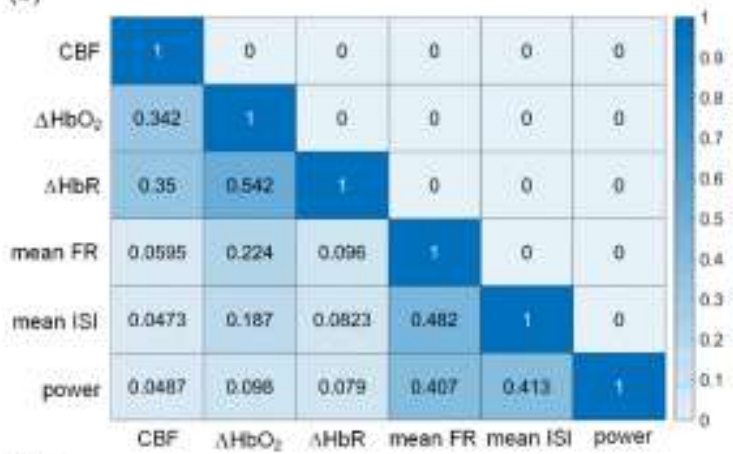

(d)

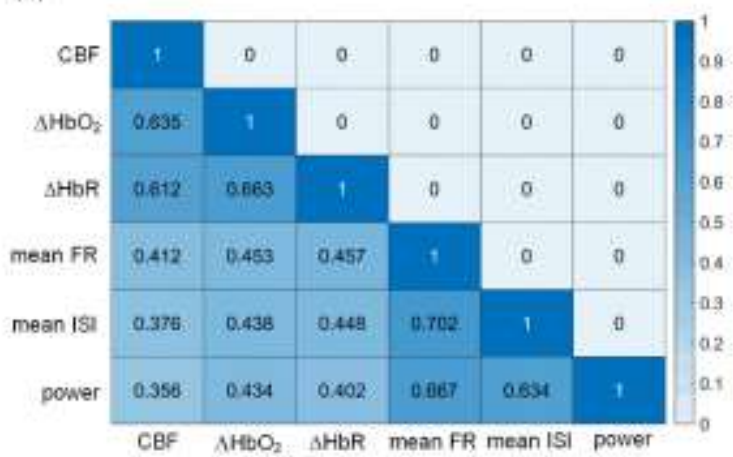

Fig. 7 determination coefficient $R^{2}$ of Pearson correlation map between hemodynamic and neuronal activity in different treatment and control conditions respectively. determination coefficient $R^{2}$ of heat map (a) within 0-120 min of G5 group, (b) within 140-220 min of G5 group, (c) within 140-220 min of G3 group, (d) within 140-220 min of G4 group Pearson, and (e) within 140-220 min of G6 group, respectively.

\section{DISCUSSION}

The purpose of the paper is to analyze the electrophysiological signals and blood oxygen signals collected by the developed optical-electrical joint system, to study the 
relationship of neural activity and hemodynamic response during the progression of LPS-induced cerebral edema, and to evaluate pharmaceutical efficacy anti-edema drugs through the correlation response.

Cerebral edema is caused by excessive infiltration and accumulation of fluid, which leads to the increase of brain volume, the essence of which is a clinical syndrome caused by the damage of blood-brain barrier and abnormal function of aquaporin, which leads to the imbalance of extracellular and extracellular water and electrolyte distribution ${ }^{16}$. At present, cerebral edema is typically classified into vasogenic edema and cytotoxic edema dependent on the pathological characteristics. Vasogenic edema is defined as extracellular accumulation of fluid resulting from disruption of the BBB, while cytotoxic edema is defined as cell swelling caused by intracellular accumulation of fluid ${ }^{17}$. Effective treatment is awaited based upon targeting the pathogenesis and chemical mediators involved in the specific edema condition. For example, those agents belonging to hypertonic macromolecular fluid would fail in treatment when BBB is completely damaged, i.e., for treating vasogenic edema, and vice versa ${ }^{18}$. However, the direct measurement of ICP could not yield sufficient information associated with the pathophysiological mechanisms, real-time and on-site monitoring of other physiological parameters, such as the hemodynamic and neuronal activity, is thus of significance for determining the specific anti-edema therapeutics acting on the different pathology ${ }^{19}$.

Previous studies have investigated the correlation of cerebral hemodynamic changes and neuronal activity. Govindan et al. combined EEG and NIRs to monitor 
neurovascular features of newborns with hypoxic ischemic encephalopathy and discussed a nonlinear model between neurovascular characteristics and disease progression ${ }^{20}$. Todd et al. used $\mathrm{fMRI}$ methods to investigate focused ultrasoundmediated disruption of BBB and found that BBB opening altered the neurovascular response in the rat brain ${ }^{21}$. Baker et al. used laser speckle imaging and endogenous optical signal imaging to study the linear relationship of cerebral blood flow, blood oxygen and metabolites in the forelimb stimulation model and found the amplitude of the functional metabolic response remained tightly coupled to the amplitude of the SEP ${ }^{22}$. Semyachkina et al. expounded the relationship between cerebral blood flow and blood-brain barrier by laser speckle imaging and optical signal imaging, indicating that the opening of blood-brain barrier is related to venous variation ${ }^{23}$. The above shows that the optical-electrical technologies can monitor the continuous brain dynamics at different spatial and temporal levels, even from microscopic changes in cell structure, ion concentration, hemodynamics, et al. In our study, it is feasible to employ the optical-electrical joint monitoring system to explore the progression of the LPS-induced cerebral edema.

LPS is one of the main pathogenic components of Gram-negative bacilli, as well as a powerful inducer of inflammation ${ }^{24}$. For example, Wang et al. found that, glial cells on striatum and cortex of rats were activated following the LPS injection, which may recapitulate the inflammatory response in cerebral hemorrhage and edema ${ }^{25}$. Numbers of studies have proved that LPS stimulated a variety of inflammatory factors, such as, IL-I $\beta$, TNF- $\alpha$, IgG, caused BBB damage, and eventually contributed to 
cerebral edema. But the pathological progression of cerebral edema induced by LPS was complex ${ }^{26}$, it is difficult to accurately monitor the pathological changes of LPS only by relying on ICP measurement, MRI or inflammatory factor immunoassay methods. In the paper, we continuously recorded the electrophysical parameters (FR, and LFPs power), and the hemodynamic parameters ( $\left.\mathrm{CBF}, \mathrm{HbO}_{2}, \mathrm{HbR}\right)$, which could capture neurovascular function with microscopic vascular and electrophysiological resolution. Our results showed that, hemodynamic and neuronal activity could reflect a physiologically complemental progress of LPS-induced by cerebral edema.

In the initial stage immediately after LPS injection, all of the monitored parameters have not demonstrated significant changes until $40 \mathrm{~min}$ in the LPS-treated group in comparison with the control group. Yet afterwards, FR, ISI, LFPs power and $\mathrm{HbO}_{2}$ started to decrease while $\mathrm{HbR}$ and $\mathrm{CBF}$ began to rise markedly from 40 min to 120 min. The time-dependent response to LPS has thus proved the correlation between the neural activity of the brain and the local cerebral blood flow. Here we inferred LPSinduced cerebral edema was highly likely to be due to changes in cerebral blood flow and blood oxygen caused by permeability destruction of the BBB.

During the late stage of LPS action, LPS might reach the brain tissue through the disruptive BBB and regulate the expression of AQP-4 in glial cells, which is dominating the process of active transport of water molecules ${ }^{27}$ and resulted in the rapid accumulation of a large amount of water and abnormal neural activity. Indeed, the expression of inflammatory mediators in the cortex associated with progressive BBB disruption was identified, and the result is consistent with hemodynamic and 
electrophysiological response.

Afterwards, two therapeutic agents, MA and HS solution, were then injected into the rats through the tail vein ${ }^{28}$. The results demonstrated that hemodynamic and electrophysiological parameters gradually recovered and the therapeutic agent indeed relieved the condition of brain edema. Futhermore, the comparison of Pearson's correlation coefficient between FR and hemodynamic parameters pre- or post- antiedema drug administration (time point: $120 \mathrm{~min}$ ) suggested that neurovascular characteristics was based on edema pathology. Thrane found that the AQP-4 increased significantly after about 100 minutes, and AQP-4 was positively correlated with cytotoxic brain edema ${ }^{29}$. Similarly, the Pearson's correlation within 140-220 min declined by more than $50 \%$ compared to that within 0-120 min in Fig.6(b). In the further research of the determination coefficient $R^{2}$, by analyzing $R^{2}$ of the $0-120$ min and 140 220 min in G5 group, the results indicated the confidence and satisfaction of the Pearson correlation between hemodynamic and neuronal activity completely changed. The pathological characteristics of LPS induced cerebral edema transformed from simple BBB permeability to water retention inside and outside nerve cells ${ }^{30}$. Due to BBB is the key to maintain the normal working environment of neurons and glial cells. by identifying the integrity of the BBB function, the neurovascular characteristic parameters are used as the basis for the evaluation of the pathological progression of cerebral edema.

\section{CONCLUSIONS}

an optical-electrical joint system was developed to study the neurovascular 
characteristic during the progression of cerebral edema and corresponding therapeutic response in a rat model using simultaneous electrophysical and hemodynamic recording. Comprehensive neurovascular and BBB information, including the FR, ISI, LFPs power, $\mathrm{CBF}, \mathrm{HbO}_{2}$, and $\mathrm{HbR}$, could be acquired and assessed concurrently to analyze neurovascular dynamic changes exhibited by the LPS-induced cerebral edema model. Our results suggested that cerebral edema may spontaneously cause blood flow disorder and neural activity abnormity due to the damage of BBB. The increased ICP by brain edema induced by LPS restricted neuronal activity, which subsequently attenuated metabolic responses and accelerated cerebral blood flow. We believe that the assessment method proposed in the present paper could provide a feasible technical solution for clinical edema pathological monitoring and drug efficacy evaluation.

\section{Ethics approval and consent to participate}

Not applicable

\section{Consent for publication}

Not applicable

\section{Availability of data and materials}

The datasets used and/or analysed during the current study are available from the corresponding author on reasonable request. All data generated or analysed during this study are included in this published article

\section{Competing interests}


All the authors declare that they have no competing interests

\section{Funding}

This work presented in the manuscript was sponsored by National Natural Science Foundation of China (61875085), National Major Scientific Instruments and Equipments Development Project Funded by National Natural Science Foundation of China (81727804), and Talent introduction research support of Nanjing Institute of Technology (YKJ202022).

\section{Authors' contributions}

The experiments were conceived and designed by Weitao Li, Yameng Zhang. Experiments were carried out by Yameng Zhang, Qian Xie and Ning Xue. Experiments data were analyzed by Yameng Zhang and Liuye Yao. The paper was written by Yameng Zhang, Yamin Yang and edited by all authors.

\section{Acknowledgements}

We thank Fan Gao for assisting with Animal perfusion and immune factor kit test.

\section{REFERENCES}

[1] Stokum J A, Kurland D B, Gerzanich V, et al. Mechanisms of Astrocyte-Mediated Cerebral Edema. Neurochem. Res. 2015; 40(2):317-328.

[2] Wykes V, Vindlacheruvu R. Intracranial pressure, cerebral blood flow and brain oedema. Surgery. 2016; 355-362.

[3] Shlosberg D, Benifla M, Kaufer D, et al. Blood-brain barrier breakdown as a therapeutic target in traumatic brain injury. Nat. Rev. Neurol. 2010; 6(7):393. 
[4] Balbi M, Koide M, Wellman G C, et al. Inversion of neurovascular coupling after subarachnoid hemorrhage in vivo. J. Cereb. Blood Flow Metab.2017; 37(11).

[5] Brad A Sutherland, Jonas C Fordsmann, Chris Martin, et al, Multi-modal assessment of neurovascular coupling during cerebral ischaemia and reperfusion using remote middle cerebral artery occlusion. J. Cereb. Blood Flow Metab. 2016; 36(4):112-115.

[6] Banks W A, Erickson M A. The blood-brain barrier and immune function and dysfunction. Neurobiol. Dis. 2010; 37(1):26-32.

[7] Kaplan L, Chow B W, Gu C. Neuronal regulation of the blood-brain barrier and neurovascular coupling. Nat Rev NeuroSci. 2020; 21(8): 416-432.

[8] Luo Z W, Ovcjak A, Wong R, et al. Drug development in targeting ion channels for brain edema. Acta Pharmacologica Sinica, 2020; 41:1272-1288.

[9] Jangula A, Murphy E J. Lipopolysaccharide-induced blood brain barrier permeability is enhanced by alpha-synuclein expression. NeuroSci Lett. 2013;551: 2327.

[10] Li w, Zhang Y, et al. Effects of puerarin on spatial learning and memory function in mice with acute alcohol consumption: An evaluation based upon firing rate and oxygen saturation analysis. ADV CLIN EXP MED: official organ Wroclaw Medical University. 2018;28(2):171-178.

[11] Zhang Y, Zhang Y M, Yu H, et al. Theta-gamma coupling in hippocampus during working memory deficits induced by low frequency electromagnetic field exposure. Physiol. Behav. 2017; 179:135-142. 
[12] Turley J.A. et al. An analysis of signal processing algorithm performance for cortical intrinsic optical signal imaging and strategies for algorithm selection. Sci. Rep. $2017 ; 7(1): 7198$.

[13] Kuboyama M, Yong F C, King T E. Studies on cytochrome oxidase. 8. Preparation and some properties of cardiac cytochrome oxidase. J. Biol. Chem.1972; 247(20):6375-6383.

[14] Zhang YM, Zhao Y, Li W, et al. Enhancement of microvessel in laser speckle image using gaussian kernel template. J Innov Opt Health Sci.12(2):1950006,2019.

[15] Endemann D H, Schiffrin E L. Endothelial dysfunction. Hospital Medicine. 1998;59(6):1983-1992.

[16] Michinaga S, Koyama Y. Pathogenesis of Brain Edema and Investigation into AntiEdema Drugs. Int. J. Mol. Sci. 2015; 16(5):9949-9975.

[17] Nag S, Manias J L, Stewart D J. Pathology and new players in the pathogenesis of brain edema. Acta Neuropathologica. 2009; 118(2):197-217.

[18] Villéga, Frédéric, Delpech J C, Griton $M$, et al. Circulating bacterial lipopolysaccharide-induced inflammation reduces flow in brain-irrigating arteries independently from cerebrovascular prostaglandin production. NeuroSci. 2017; 346:160-172.

[19] Michinaga S, Koyama Y. Pathogenesis of Brain Edema and Investigation into AntiEdema Drugs. Int. J. Mol. Sci. 2015;16(5):9949-9975.

[20] Govindan R B, Massaro A, Chang T, et al. A novel technique for quantitative bedside monitoring of neurovascular coupling. J. Neurosci. Methods. 2015; 259:135- 
142.

[21] Todd N, Zhang Y, Livingstone $\mathrm{M}$, et al. The neurovascular response is attenuated by focused ultrasound-mediated disruption of the blood-brain barrier. Neurolmage. 2019; 201:116-123.

[22] Baker W B, Sun Z, Hiraki T, et al. Neurovascular coupling varies with level of global cerebral ischemia in a rat model. J Cereb Blood Flow Metab. 2013; 33(1):97105.

[23] Semyachkina-Glushkovskaya S, Abdurashitov A, Pavlov A, et al. Laser speckle imaging and wavelet analysis of cerebral blood flow associated with the opening of the blood-brain barrier by sound. Chinese Opt Let. 2017 15(9):2-6,.

[24] Koh H, Tasaka S, Hasegawa N, et al. Protective role of vascular endothelial growth factor in endotoxin-induced acute lung injury in mice. Respir. Res. 2007; 8(1):60-62. [25] Wang X, Rousset C I, Hagberg H, et al. Lipopolysaccharide-induced inflammation and perinatal brain injury. Semin Fetal Neonatal Med. 2006; 11(5):343-353.

[26] Cai Z, Pang Y Lin S, et a1. Differential roles of tumor necrosis factor-alpha and interleukin-1 beta in lipopolysaccharide-induced brain injury in the neonatal rat. Brain Res.2003; 975(1-2): 37-47.

[27] $\mathrm{Y}$ Liu, Wang L, Du $\mathrm{N}$, et al. Ramelteon Ameliorates LPS-Induced Hyperpermeability of the Blood-Brain Barrier (BBB) by Activating Nrf2. Inflammation. $2021 ; 4$

[28] Ballocco I, Evangelisti M A, Deiana R, et al. A pilot study evaluating the effect of mannitol and hypertonic saline solution in the treatment of increased intracranial 
pressure in 2 cats and 1 dog naturally affected by traumatic brain injury. $\mathrm{J}$ Vet Emerg Crit Care. 2019; 29(5): 578-584.

[29] Thrane, A. S., Rappold, P. M., et al. Critical role of aquaporin-4 (AQP4) in astrocytic Ca2+ signaling events elicited by cerebral edema. PNAS. 2011; 108(2): 846-851.

[30] Wu J, Ding D, Wang X, et al. Regulation of aquaporin 4 expression by lipoxin A4 in astrocytes stimulated by lipopolysaccharide. Cel Imm. 2019; 344:159-163. 